\title{
Automorphisms and some geodesic properties of ortho-Grassmann graphs
}

\author{
Mark Pankov \\ Faculty of Mathematics and Computer Science \\ University of Warmia and Mazury \\ Słoneczna 54, 10-710 Olsztyn, Poland \\ pankov@matman.uwm.edu.pl \\ Krzysztof Petelczyc Mariusz Żynel \\ Faculty of Mathematics \\ University of Białystok \\ Ciołkowskiego 1M, 15-245 Białystok, Poland \\ $\{$ kryzpet, mariusz\}@math.uwb.edu.pl
}

Submitted: Mar 9, 2021; Accepted: Nov 26, 2021; Published: Dec 17, 2021

(C) The authors. Released under the CC BY-ND license (International 4.0).

\begin{abstract}
Let $H$ be a complex Hilbert space. Consider the ortho-Grassmann graph $\Gamma_{k}^{\perp}(H)$ whose vertices are $k$-dimensional subspaces of $H$ (projections of rank $k$ ) and two subspaces are connected by an edge in this graph if they are compatible and adjacent (the corresponding rank- $k$ projections commute and their difference is an operator of rank 2). Our main result is the following: if $\operatorname{dim} H \neq 2 k$, then every automorphism of $\Gamma_{k}^{\perp}(H)$ is induced by a unitary or anti-unitary operator; if $\operatorname{dim} H=2 k \geqslant 6$, then every automorphism of $\Gamma_{k}^{\perp}(H)$ is induced by a unitary or anti-unitary operator or it is the composition of such an automorphism and the orthocomplementary map. For the case when $\operatorname{dim} H=2 k=4$ the statement fails. To prove this statement we compare geodesics of length two in ortho-Grassmann graphs and characterise compatibility (commutativity) in terms of geodesics in Grassmann and ortho-Grassmann graphs. At the end, we extend this result on generalised ortho-Grassmann graphs associated to conjugacy classes of finite-rank self-adjoint operators.
\end{abstract}

Mathematics Subject Classifications: 05E18, 47B15, 81P10 


\section{Introduction}

Classic Chow's theorem [1] states that all automorphisms of Grassmann graphs can be obtained from semi-linear automorphisms of the associated vector spaces or semi-linear isomorphisms to the dual vector spaces. Recall that two vertices in the Grassmann graph formed by $k$-dimensional subspaces are connected by an edge if the corresponding subspaces are adjacent, i.e. their intersection is $(k-1)$-dimensional. There are results in spirit of Chow's theorem concerning automorphisms of polar Grassmann graphs $[2,8]$ and analogues for matrix spaces [14]. Methods used to prove Chow-type theorems are based on a description of maximal cliques and their intersections in the corresponding graph.

Recently, Chow's theorem was successfully exploited in the discipline known as preserver problems related to quantum mechanics which provides various characterisations of quantum symmetries, i.e. unitary and anti-unitary operators [3, 4, 9]. The Grassmannian formed by $k$-dimensional subspaces of a complex Hilbert space $H$ can be identified with the conjugacy class of rank- $k$ projections and two such projections are connected by an edge in the associated Grassmann graph if their difference is an operator of rank 2 (i.e. the smallest possible). The automorphisms of such Grassmann graphs cannot be characterised as the transformations induced by unitary and anti-unitary operators and we look for a modification of this graph which will satisfy that condition. Our choice is so-called ortho-Grassmann graph, where two subspaces are connected by an edge if they are adjacent and compatible. Then the corresponding projections commute and, as in the Grassmann graph, their difference is an operator of rank 2.

Recall that projections can be characterised as self-adjoint idempotents in the algebra of bounded operators. Observables in quantum mechanics are identified with (not necessarily bounded) self-adjoint operators and two bounded observables are simultaneously testable if and only if the corresponding bounded self-adjoint operators commute (see, for example, [13, Theorem 4.11]). So, our ortho-adjacency relation is naturally interpreted in terms of quantum mechanics.

The main result (Theorem 3) states that every automorphism of the ortho-Grassmann graph can be obtained from a unitary or anti-unitary operator except the case when the graph consists of 2-dimensional subspaces and $\operatorname{dim} H=4$. A simple example shows that the statement fails for this case (Example 5).

In [5] the same statement is proved for the graph formed by 2-dimensional subspaces of a vector space with an anisotropic symmetric form. Also, [5] contains some deep results concerning the hard case when the dimension of the vector space is 4 .

There is an analogue of our result for the set of all non-isotropic $k$-dimensional subspaces of a sesquilinear form under the assumption that the dimension of the associated vector space is not equal to $2 k$ [11]. The method exploited in [11] (a description of maximal cliques and their intersections) does not work for the case when the graph is formed by subspaces whose dimension is half of $\operatorname{dim} H$ (Section 3.3) and thus we use completely different reasonings. Our proof is based on the comparison of geodesics of length two in ortho-Grassmann graphs (Section 4) and a characterisation of compatibility (commutativity) in terms of geodesics in Grassmann and ortho-Grassmann graphs (Theorem 10). 
In quantum mechanics, rank-one projections, and consequently, 1-dimensional subspaces are identified with pure states [13, Theorem 4.23]. By classic Wigner's theorem, all symmetries of pure states are induced by unitary or anti-unitary operators. Observe that two 1-dimensional subspaces are ortho-adjacent if and only if they are orthogonal, i.e. for the Grassmannian of 1-dimensional subspaces the ortho-adjacency and orthogonality relations coincide; in this case, our result is Uhlhorn's version of Wigner's theorem [12]. In the general case, the ortho-adjacency relation can be characterised in terms of principal angles as follows: two subspaces are ortho-adjacent if and only if the principal angles between them are $0, \ldots, 0, \pi / 2$ (see, for example, [9, Section 4.4]). Transformations (not necessarily bijective) of Hilbert Grassmannians preserving the principal angles between any pair of subspaces are described in $[6,7]$. Our result provides a description of bijections which preserve principal angles of type $0, \ldots, 0, \pi / 2$.

There is an analogue of Chow's theorem for conjugacy classes of finite-rank self-adjoint operators [10]. Using this statement and results from Section 4 we extend the main result on such classes of operators (Theorem 25).

\section{Main result}

Let $V$ be a vector space over a field $F$. For every integer $k$ satisfying $0<k<\operatorname{dim} H$ (if $H$ is infinite-dimensional, then for every integer $k>0$ ) we denote by $\mathcal{G}_{k}(V)$ the Grassmannian formed by $k$-dimensional subspaces of $V$. Two $k$-dimensional subspaces of $V$ are called adjacent if their intersection is $(k-1)$-dimensional, or equivalently, their sum is $(k+1)$-dimensional. In the case when $k=1$ or $\operatorname{dim} V=k+1$, any two distinct elements of $\mathcal{G}_{k}(V)$ are adjacent. The Grassmann graph $\Gamma_{k}(V)$ is the simple graph whose vertex set is $\mathcal{G}_{k}(V)$ and two $k$-dimensional subspaces of $V$ are connected in this graph by an edge if they are adjacent.

Recall that a semi-linear automorphism of $V$ is a bijection $L: V \rightarrow V$ such that

$$
L(x+y)=L(x)+L(y)
$$

for all $x, y \in V$ and there is an automorphism $\sigma$ of the field $F$ satisfying

$$
L(a x)=\sigma(a) L(x)
$$

for all $a \in F$ and $x \in V$. Every semi-linear automorphism of $V$ induces an automorphism of $\Gamma_{k}(V)$. If $\operatorname{dim} V=2 k$ and $L: V \rightarrow V^{*}$ is a semi-linear isomorphism, then the map sending every $k$-dimensional subspace $X \subset V$ to $L(X)^{0}$ is an automorphism of $\Gamma_{k}(V)$ (for a subspace $Y$ of the dual vector space $V^{*}$ the annihilator $Y^{0}$ is formed by all $x \in V$ satisfying $y^{*} x=0$ for all $y^{*} \in Y$ and the dimension of $Y^{0}$ is equal to the codimension of $Y)$.

Theorem 1 (Chow [1]). Suppose that $k$ is an integer satisfying $1<k<\operatorname{dim} V-1$ (if $V$ is infinite-dimensional, then $k$ is an arbitrary integer greater than 1 ). Then every automorphism of $\Gamma_{k}(V)$ is induced by a semi-linear automorphism of $V$ or a semi-linear isomorphism of $V$ to $V^{*}$ and the second possibility is realised only in the case when $\operatorname{dim} V=2 k$. 
Remark 2. In [1] this statement was obtained for finite-dimensional vector spaces only; but it holds for the general case; see, for example, [9, Section 2.4].

Let $H$ be a complex Hilbert space of dimension not less than 3. Closed subspaces of $H$ can be naturally identified with projections, i.e. self-adjoint idempotents in the algebra of all bounded operators. A closed subspace $X \subset H$ corresponds to the projection $P_{X}$ whose image is $X$. Then $\mathcal{G}_{k}(H)$ coincides with the conjugacy class of rank- $k$ projections $\mathcal{P}_{k}(H)$. Two $k$-dimensional subspaces $X, Y \subset H$ are adjacent if and only if the difference of the corresponding projections $P_{X}-P_{Y}$ is of rank 2, i.e. of minimal rank (the difference of two self-adjoint operators from the same conjugacy class cannot be of rank 1). Two closed subspaces $X, Y \subset H$ are called compatible if there is an orthonormal basis of $H$ such that $X$ and $Y$ are spanned by subsets of this basis; this is equivalent to the fact that the projections $P_{X}$ and $P_{Y}$ commute. We say that $k$-dimensional subspaces $X, Y \subset H$ are ortho-adjacent if they are adjacent and compatible, i.e.

$$
\operatorname{rank}\left(P_{X}-P_{Y}\right)=2 \quad \text { and } \quad P_{X} P_{Y}=P_{Y} P_{X}
$$

Denote by $\Gamma_{k}^{\perp}(H)$ the simple graph whose vertex set is $\mathcal{G}_{k}(H)$ and two $k$-dimensional subspaces of $H$ are connected by an edge in this graph if they are ortho-adjacent. Then $\Gamma_{k}^{\perp}(H)$ can be considered as the graph whose vertex set is $\mathcal{P}_{k}(H)$ and two rank- $k$ projections $P_{X}, P_{Y}$ are connected by an edge if the conditions (1) are satisfied. In contrast to the Grassmann graph $\Gamma_{k}(H)$, for $k=1$ or $\operatorname{dim} H=k+1$ the graph $\Gamma_{k}^{\perp}(H)$ contains pairs of distinct vertices which are not connected by an edge. In the next section, we show that this graph is connected.

An invertible linear operator on $H$ is unitary if it preserves the inner product. An invertible conjugate-linear operator $U$ on $H$ (i.e. satisfying $U(x+y)=U(x)+U(y)$ and $U(a x)=\bar{a} U(x)$ for all $x, y \in H$ and all scalars $a)$ is called anti-unitary if

$$
\langle U(x), U(y)\rangle=\overline{\langle x, y\rangle}
$$

for all $x, y \in H$. Unitary and anti-unitary operators induce automorphisms of the graph $\Gamma_{k}^{\perp}(H)$. If $\operatorname{dim} H=n$ is finite, then the orthocomplementary map sending every $k$ dimensional subspace $X \subset H$ to the orthogonal complement $X^{\perp}$ is an isomorphism between $\Gamma_{k}^{\perp}(H)$ and $\Gamma_{n-k}^{\perp}(H)$.

Our main result is the following.

Theorem 3. If $\operatorname{dim} H \neq 2 k$ (in particular, if $H$ is infinite-dimensional), then every automorphism of the graph $\Gamma_{k}^{\perp}(H)$ is induced by a unitary or anti-unitary operator. In the case when $\operatorname{dim} H=2 k \geqslant 6$, every automorphism of $\Gamma_{k}^{\perp}(H)$ is induced by a unitary or anti-unitary operator or it is the composition of such an automorphism and the orthocomplementary map.

Remark 4. If $X$ is a $k$-dimensional subspace of $H$, then $P_{X^{\perp}}=\mathrm{Id}-P_{X}$ and

$$
P_{U(X)}=U P_{X} U^{*}
$$


for every unitary or anti-unitary operator $U$ on $H$. Consider $\Gamma_{k}^{\perp}(H)$ as the graph whose vertices are rank- $k$ projections. Then Theorem 3 can be reformulated as follows. If $\operatorname{dim} H \neq 2 k$ and $f$ is an automorphism of $\Gamma_{k}^{\perp}(H)$, then there is a unitary or anti-unitary operator $U$ on $H$ such that

$$
f(P)=U P U^{*}
$$

for all $P \in \mathcal{P}_{k}(H)$. If $\operatorname{dim} H=2 k \geqslant 6$ and $f$ is an automorphism of $\Gamma_{k}^{\perp}(H)$, then there is a unitary or anti-unitary operator $U$ on $H$ such that (2) holds for all $P \in \mathcal{P}_{k}(H)$ or we have

$$
f(P)=U(\operatorname{Id}-P) U^{*}
$$

for every $P \in \mathcal{P}_{k}(H)$.

The above statement fails if $\operatorname{dim} H=2 k=4$, see Example 5 .

Two 1-dimensional subspaces of $H$ are ortho-adjacent if and only if they are orthogonal. So, Theorem 3 coincides with Uhlhorn's version of Wigner's theorem concerning bijective transformations of $\mathcal{G}_{1}(H)$ preserving the orthogonality relation in both directions [12]. Therefore, it is sufficient to prove Theorem 3 only for the case when $4 \leqslant 2 k \leqslant \operatorname{dim} H$. Indeed, if $\operatorname{dim} H=n$ is finite and $2 k>n$, then for every automorphism $f$ of $\Gamma_{k}^{\perp}(H)$ the map sending every $(n-k)$-dimensional subspace $X \subset H$ to $\left(f\left(X^{\perp}\right)\right)^{\perp}$ is an automorphism of $\Gamma_{n-k}^{\perp}(H)$; if this automorphism is induced by a unitary or anti-unitary operator, then $f$ is induced by the same operator.

Our proof of Theorem 3 is based on a characterisation of adjacency in terms of orthoadjacency (Section 4) which immediately implies that every automorphism $f$ of $\Gamma_{k}^{\perp}(H)$ is an automorphism of $\Gamma_{k}(H)$ (except the case when $\operatorname{dim} H=2 k=4$ ). It follows from Theorem 1 that there is a semi-linear automorphism $L: H \rightarrow H$ such that one of the following possibilities is realised:

- $f(X)=L(X)$ for every $k$-dimensional subspace $X \subset H$,

- $\operatorname{dim} H=2 k$ and $f(X)=L(X)^{\perp}$ for every $k$-dimensional subspace $X \subset H$.

We cannot immediately assert that $L$ is a scalar multiple of a unitary or anti-unitary operator. This follows from our characterisation of compatibility in terms of geodesics in the graphs $\Gamma_{k}^{\perp}(H)$ and $\Gamma_{k}(H)$ (Theorem 10).

Example 5. Suppose that $\operatorname{dim} H=4$. Then for any ortho-adjacent 2-dimensional subspaces $X, Y \subset H$ the subspaces $X, Y^{\perp}$ are also ortho-adjacent (we take any orthonormal basis of $H$ whose subsets span $X, Y$ and observe that $Y^{\perp}$ also is spanned by a subset of this basis). Let us fix a certain 2-dimensional subspace $X \subset H$ and consider the transformation of $\mathcal{G}_{2}(H)$ which transposes $X, X^{\perp}$ and leaves fixed all other 2-dimensional subspaces of $H$. This is an automorphism of $\Gamma_{2}^{\perp}(H)$. More generally, we can take any subset $\mathcal{X} \subset \mathcal{G}_{2}(H)$ such that $X^{\perp} \in \mathcal{X}$ for all $X \in \mathcal{X}$ and the transformation of $\mathcal{G}_{2}(H)$ which sends all $X \in \mathcal{X}$ to $X^{\perp}$ and leaves all elements of $\mathcal{G}_{2}(H) \backslash \mathcal{X}$ fixed. As above, we obtain an automorphism of $\Gamma_{2}^{\perp}(H)$. Our conjecture is the following: if $\operatorname{dim} H=4$ and $f$ is an automorphism of $\Gamma_{2}^{\perp}(H)$, then there is a unitary or anti-unitary operator $U$ such that for every 2-dimensional subspace $X \subset H$ we have $f(X)=U(X)$ or $f(X)=U(X)^{\perp}$. 


\section{Grassmann graphs and ortho-Grassmann graphs}

\subsection{Some elementary properties}

Recall that the distance $d(v, w)$ between two vertices $v, w$ in a connected simple graph is defined as the smallest number $m$ such that there is a path of length $m$ (i.e. a path which consists of $m$ edges) connecting these vertices. A path connecting $v$ and $w$ is called a geodesic if its length is $d(v, w)$.

We will use some basic properties of the distance in Grassmann graphs (see, for example, [9, Section 2.3]). The Grassmann graph $\Gamma_{k}(H)$ is connected; furthermore, for any $k$-dimensional subspaces $X, Y \subset H$ the following assertions are fulfilled:

(D1) the distance between $X$ and $Y$ in $\Gamma_{k}(H)$ is equal to $k-\operatorname{dim}(X \cap Y)$;

(D2) every $k$-dimensional subspace in a geodesic of $\Gamma_{k}(H)$ connecting $X$ with $Y$ contains $X \cap Y$ and is contained in $X+Y$;

(D3) if the distance between $X$ and $Y$ is equal to $m$ and $Z$ is a $k$-dimensional subspace of $H$ such that the distances from $Z$ to $X$ and $Y$ are equal to $i$ and $m-i$ (respectively), then there is a geodesic in $\Gamma_{k}(H)$ connecting $X$ with $Y$ and containing $Z$.

If $X, Y, Z$ are mutually adjacent $k$-dimensional subspaces of $H$, then at least one of the following possibilities is realised:

(T1) $X \cap Y \cap Z$ is $(k-1)$-dimensional,

(T2) $X+Y+Z$ is $(k+1)$-dimensional.

Note that there are triples of $k$-dimensional subspaces $X, Y, Z$ satisfying both (T1) and (T2). In the case when (T1) fails and (T2) holds, $X \cap Y \cap Z$ is $(k-2)$-dimensional and $k>1$. If (T1) holds and (T2) fails, then $X+Y+Z$ is $(k+2)$-dimensional and $k<\operatorname{dim} H-1$. In the case when $1<k<\operatorname{dim} H-1$, there are precisely two types of maximal cliques in $\Gamma_{k}(H)$ :

- the $\operatorname{star} \mathcal{S}(S), S \in \mathcal{G}_{k-1}(H)$, consisting of all $k$-dimensional subspaces containing $S$;

- the top $\mathcal{G}_{k}(U), U \in \mathcal{G}_{k+1}(H)$.

Every automorphism of $\Gamma_{k}(H)$ preserves types of maximal cliques (stars go to stars and tops go to tops) or it sends all stars to tops and all tops to stars. The first possibility is realised if and only if this automorphism is induced by a semi-linear automorphism of $H$. See $[9$, Section 2.4] for the details.

Now, we describe all possible connections in $\Gamma_{k}^{\perp}(H)$ between two non-compatible adjacent $k$-dimension subspaces.

Lemma 6. Let $X, Y$ be adjacent $k$-dimensional subspaces of $H$ and $k<\operatorname{dim} H-1$. $A k$-dimensional subspace $Z \not \subset X+Y$ is ortho-adjacent to both $X, Y$ if and only if $Z=(X \cap Y)+P$ for some 1-dimensional subspace $P$ orthogonal to $X+Y$. 
Proof. The condition $k<\operatorname{dim} H-1$ implies that $X+Y$ is a proper subspace of $H$. For every 1-dimensional subspace $P \subset(X+Y)^{\perp}$ the $k$-dimensional subspace $P+(X \cap Y)$ is ortho-adjacent to both $X, Y$. Conversely, if $Z$ is a $k$-dimensional subspace ortho-adjacent to both $X, Y$ and $Z \not \subset X+Y$, then $Z$ contains $X \cap Y$ as a hyperplane (the case (T1)) and the 1-dimensional orthogonal complement of $X \cap Y$ in $Z$ is orthogonal to both $X, Y$, and consequently, to $X+Y$.

Lemma 7. Let $X, Y$ be adjacent $k$-dimensional subspaces of $H$ with $k>1$ and let $S$ be the 2-dimensional orthogonal complement of $X \cap Y$ in $X+Y$. A $k$-dimensional subspace $Z \subset X+Y$ is ortho-adjacent to both $X, Y$ if and only if $Z=S+W$ for some $(k-2)$ dimensional subspace $W \subset X \cap Y$.

Proof. For every $(k-2)$-dimension subspace $W \subset X \cap Y$ the $k$-dimensional subspace $W+S$ is ortho-adjacent to both $X, Y$. Indeed, $X, Y, W+S$ are hyperplanes in $X+Y$ and $W+S$ contains the 1-dimensional subspaces

$$
X^{\perp} \cap(X+Y) \text { and } Y^{\perp} \cap(X+Y)
$$

orthogonal to $X$ and $Y$, respectively. If a $k$-dimensional subspace $Z \subset X+Y$ is orthoadjacent to both $X, Y$, then it contains the 1-dimensional subspaces (3), and consequently, their sum $S$. This implies that $Z$ intersects $X \cap Y$ in a $(k-2)$-dimensional subspace.

Proposition 8. The graph $\Gamma_{k}^{\perp}(H)$ is connected.

Proof. By Lemmas 6 and 7, for any two non-compatible adjacent $k$-dimensional subspaces of $H$ there is a $k$-dimensional subspace ortho-adjacent to each of them. The required statement is a consequence of the fact that the Grassmann graph $\Gamma_{k}(H)$ is connected.

The distance between two non-compatible adjacent $k$-dimensional subspaces of $H$ in $\Gamma_{k}^{\perp}(H)$ is equal to 2 . The following observation concerns the number of geodesics in $\Gamma_{k}^{\perp}(H)$ connecting such subspaces.

Lemma 9. If $k$-dimensional subspaces $X, Y \subset H$ are adjacent and non-compatible, then the number of geodesics in $\Gamma_{k}^{\perp}(H)$ connecting $X$ and $Y$ is infinite except the case when $\operatorname{dim} H=2 k=4$. In this exceptional case, there are precisely 2 such geodesics.

Proof. If $Z$ is a $k$-dimensional subspace ortho-adjacent to both $X, Y$, then one of the following possibilities is realised:

(1) $k<\operatorname{dim} H-1$ and $Z$ is the sum of $X \cap Y$ and a 1-dimensional subspace orthogonal to $X+Y$,

(2) $k>1$ and $Z$ is the sum of a $(k-2)$-dimensional subspace of $X \cap Y$ and the 2dimensional orthogonal complement of $X \cap Y$ in $X+Y$.

If $\operatorname{dim} H=2 k=4$, then there is precisely one $Z$ satisfying (1) and precisely one satisfying (2). For the remaining cases there are infinitely many $Z$ satisfying at least one of the conditions (1), (2). 


\subsection{A characterisation of compatibility in terms of geodesics}

If $X, Y$ are compatible $k$-dimensional subspaces of $H$, then every geodesic of $\Gamma_{k}(H)$ connecting $X$ and $Y$ is a geodesic in $\Gamma_{k}^{\perp}(H)$; furthermore, any two $k$-dimensional subspaces in such a geodesic are compatible; see [9, Lemma 4.31].

Theorem 10. Two $k$-dimensional subspaces $X, Y \subset H$ are compatible if and only if every geodesic of $\Gamma_{k}(H)$ connecting $X$ and $Y$ is a geodesic in $\Gamma_{k}^{\perp}(H)$.

Proof. By [9, Lemma 4.31], we have to prove the following: if every geodesic of $\Gamma_{k}(H)$ connecting $X$ and $Y$ is a geodesic in $\Gamma_{k}^{\perp}(H)$, then $X, Y$ are compatible.

We start from the case when $X \cap Y=0$, i.e. the distance between $X$ and $Y$ in $\Gamma_{k}(H)$ is equal to $k$ (the property (D1)). We need to show that $X$ and $Y$ are orthogonal. The subspace $X+Y$ is $2 k$-dimensional and we denote by $X^{\prime}$ the $k$-dimensional subspace which is the orthogonal complement of $X$ in $X+Y$. Let us take any 1-dimensional subspace $P \subset Y$ and a $(k-1)$-dimensional subspace $N \subset X$. The $k$-dimensional subspace $P+N$ is adjacent to $X$ and the distance between $P+N$ and $Y$ in $\Gamma_{k}(H)$ is equal to $k-1$ (the property (D1)). By the property (D3), there is a geodesic of $\Gamma_{k}(H)$ which connects $X$ with $Y$ and contains $P+N$. By our assumption, this is a geodesic in $\Gamma_{k}^{\perp}(H)$ and $P+N$ is ortho-adjacent to $X$. Therefore, $P+N$ contains a 1-dimensional subspace $Q$ orthogonal to $X$, in other words, $P+N$ intersects $X^{\prime}$ precisely in $Q$. Suppose that $Q$ is distinct from $P$. We choose a $(k-1)$-dimensional subspace $M \subset X$ such that $P+M$ does not contain $Q$ (such subspace exists, since the intersection of all $P+N$, where $N$ is a $(k-1)$-dimensional subspace of $X$, coincides with $P$ and $P \neq Q$ by assumption). Then $P+M$ intersects $X^{\prime}$ in a 1-dimensional subspace $Q^{\prime}$ distinct from $Q$. The 2-dimensional subspace $Q^{\prime}+Q$ is contained in the intersection of $X^{\prime}$ and $P+X$. Since $X$ is a hyperplane of $P+X$, the subspace $Q^{\prime}+Q$ has a non-zero intersection with $X$ which is impossible. This contradiction means that $P=Q$. So, we have established that every 1-dimensional subspace $P \subset Y$ is contained in $X^{\prime}$. Therefore, $Y=X^{\prime}$, and consequently, $X$ is orthogonal to $Y$.

Suppose that $\operatorname{dim}(X \cap Y)=m$ and $0<m<k-1$ (the case when $X$ and $Y$ are adjacent is trivial). Then

$$
\operatorname{dim}(X+Y)=2 k-m
$$

and the distance between $X$ and $Y$ in $\Gamma_{k}(H)$ is equal to $k-m$ (the property (D1)). Denote by $V$ the orthogonal complement of $X \cap Y$ in $X+Y$. The dimension of this subspace is equal to $2(k-m)$. The intersection of the $(k-m)$-dimensional subspaces

$$
X^{\prime}=X \cap V \quad \text { and } \quad Y^{\prime}=Y \cap V
$$

is zero and the distance between them in $\Gamma_{k-m}(H)$ is equal to $k-m$. If

$$
X^{\prime}=Z_{0}^{\prime}, Z_{1}^{\prime}, \ldots, Z_{k-m}^{\prime}=Y^{\prime}
$$

is a geodesic in $\Gamma_{k-m}(H)$, then each $Z_{i}^{\prime}$ is contained in $V$ (the property (D2)) and

$$
X=Z_{0}, Z_{1}, \ldots, Z_{k-m}=Y \quad \text { with } \quad Z_{i}=Z_{i}^{\prime}+(X \cap Y), i \in\{0,1, \ldots, k-m\}
$$


is a geodesic in $\Gamma_{k}(H)$. By our assumption, (5) is a geodesic in $\Gamma_{k}^{\perp}(H)$ which implies that $Z_{i-1}$ and $Z_{i}$ are ortho-adjacent for every $i \in\{1, \ldots, k-m\}$. The latter guarantees that $Z_{i-1}^{\prime}$ and $Z_{i}^{\prime}$ are ortho-adjacent for every $i \in\{1, \ldots, k-m\}$, i.e. (4) is a geodesic in $\Gamma_{k-m}^{\perp}(H)$. So, every geodesic of $\Gamma_{k-m}(H)$ connecting $X^{\prime}$ and $Y^{\prime}$ is a geodesic in $\Gamma_{k-m}^{\perp}(H)$. Applying arguments from the previous paragraph, we establish that $X^{\prime}$ and $Y^{\prime}$ are orthogonal. This means that $X$ and $Y$ are compatible.

As an application of Theorem 10, we prove the following statement which will be exploited to prove Theorem 3 .

Lemma 11. Suppose that $4 \leqslant 2 k \leqslant \operatorname{dim} H$ and $f$ is a bijective transformation of $\mathcal{G}_{k}(H)$ which is an automorphism of both $\Gamma_{k}(H)$ and $\Gamma_{k}^{\perp}(H)$. Then $f$ is induced by a unitary or anti-unitary operator or it is the composition of a bijection induced by a unitary or anti-unitary operator and the orthocomplementary map. The second possibility is realised only in the case when $\operatorname{dim} H=2 k$.

Proof. If $f$ sends stars to stars and tops to tops, then $f$ is induced by a semi-linear automorphism of $H$; otherwise, this property holds for the composition of $f$ and the orthocomplementary map (since the orthocomplementation transfers stars to tops and tops to stars). Therefore, there is a semilinear automorphism $U$ of $H$ such that one of the following possibilities is realised:

- $f(X)=U(X)$ for all $X \in \mathcal{G}_{k}(H)$,

- $\operatorname{dim} H=2 k$ and $f(X)=U(X)^{\perp}$ for all $X \in \mathcal{G}_{k}(H)$.

We need to show that $U$ is a scalar multiple of a unitary or anti-unitary operator (since $U$ and $a U$ induce the same transformation of $\mathcal{G}_{k}(H)$ for every non-zero scalar $a$ ). It is sufficient to consider the first case (in the second case, we replace $f$ by the composition of $f$ and the orthocomplementary). Since $f$ is an automorphism of both $\Gamma_{k}^{\perp}(H)$ and $\Gamma_{k}(H)$, Theorem 10 guarantees that $f$ is compatibility preserving in both directions. Two $k$-dimensional subspaces of $H$ are orthogonal if and only if they are compatible and the distance between them in $\Gamma_{k}(H)$ is equal to $k$. This means that $f$ is orthogonality preserving in both directions and $U$ sends orthogonal vectors to orthogonal vectors which implies that $U$ is a scalar multiple of a unitary or anti-unitary operator [9, Proposition $4.2]$.

\subsection{Remarks}

It was pointed out above that it is sufficient to prove Theorem 3 only for the case when $4 \leqslant 2 k \leqslant \operatorname{dim} H$. If $\operatorname{dim} H \neq 2 k$, then Theorem 3 is a direct consequence of Lemma 11 and the following statement.

Lemma 12 (Lemma 4.37 in [9]). If $2<2 k<\operatorname{dim} H$, then every ortho-adjacency preserving injective transformation of $\mathcal{G}_{k}(H)$ is adjacency preserving.

Remark 13. For $k=2$ Lemma 12 is proved in [5]. 
In [11], an analogue of Theorem 3 is proved for the set of all non-isotropic $k$-dimensional subspaces of a sesquilinear form under the assumption that the dimension of the associated vector space is distinct from $2 k$. Methods used to prove Lemma 12 as well as methods exploited in [11] are based on some properties of maximal cliques in ortho-Grassmann graphs.

Every maximal clique of $\Gamma_{k}^{\perp}(H)$ is the intersection of a star or a top (a maximal clique of $\left.\Gamma_{k}(H)\right)$ with the set of all $k$-dimensional subspaces spanned by subsets of an orthonormal basis of $H$ such that the $(k-1)$-dimensional or $(k+1)$-dimensional subspace associated with the star or the top (respectively) is also spanned by a subset of this basis [9, Section 4.6]. Such a clique will be called an ortho-star or an ortho-top, respectively. Every ortho-top contains precisely $k+1$ elements; an ortho-star contains precisely $n-k+1$ elements if $\operatorname{dim} H=n$ is finite, and it is infinite if $H$ is infinite-dimensional [9, Lemma 4.30]. In this way ortho-stars can be easily distinguished from ortho-tops, but not in the case when $\operatorname{dim} H=2 k$ and they have the same number of elements.

If $2<2 k<\operatorname{dim} H$, then every ortho-adjacency preserving injective transformation of $\mathcal{G}_{k}(H)$ sends ortho-stars to subsets of ortho-stars. This observation is a crucial tool in the proof of Lemma 12. Similarly, transformations considered in [11] send ortho-stars to ortho-stars and ortho-tops to ortho-tops.

It was conjectured in [11] that the method used to prove Chow's theorem can be modified for the case when $\operatorname{dim} H=2 k$. The key argument is the following: if the intersection of a star and a top is non-empty, then the number of elements in this intersection is greater than the number of elements in the intersection of two distinct stars or the intersection of two distinct tops. Consequently, if one star goes to a top under an automorphism of the Grassmann graph, then all stars go to tops and all tops go to stars.

Now, we explain why the same arguments do not work for ortho-stars and ortho-tops in the case when $\operatorname{dim} H=2 k$. The intersection of two distinct maximal cliques of $\Gamma_{k}^{\perp}(H)$ depends on the relations between the associated $(k-1)$-dimensional or $(k+1)$-dimensional subspaces and orthonormal bases. A direct verification shows that the following assertions are fulfilled:

- The number of elements in the intersection of two distinct ortho-stars associated to the same $(k-1)$-dimensional subspace can take any value $m \in\{0,1, \ldots, k-1\}$ and the same holds for the intersection of two distinct ortho-tops corresponding to the same $(k+1)$-dimensional subspace.

- The intersection of two ortho-stars associated to distinct $(k-1)$-dimensional subspaces is empty or consists of one element and the same holds for the intersection of two ortho-tops corresponding to distinct $(k+1)$-dimensional subspaces.

- The number of elements in the intersection of an ortho-star and an ortho-top can take any value $m \in\{0,1,2\}$.

Consider ortho-stars $\mathcal{S}_{1}, \mathcal{S}_{2}$ associated to distinct $(k-1)$-dimensional subspaces and such that $\mathcal{S}_{1} \cap \mathcal{S}_{2}$ is one element. If $\mathcal{S}_{1}$ goes to an ortho-top $\mathcal{T}$ under an automorphism of $\Gamma_{k}^{\perp}(H)$, then this automorphism sends $\mathcal{S}_{2}$ to an ortho-star or to an ortho-top intersecting 
$\mathcal{T}$ precisely in one element. Therefore, to claim that an automorphism of $\Gamma_{k}^{\perp}(H)$ either preserves or exchanges two types of maximal cliques, we need different arguments.

Our proof of Theorem 3 is based on a characterisation of adjacency in terms of orthoadjacency and do not depend on the relation between $\operatorname{dim} H$ and $k$.

\section{A characterization of adjacency in terms of ortho-adjacency and proof of Theorem 3}

In this section, we investigate geodesics of length 2 in the ortho-Grassmann graph $\Gamma_{k}^{\perp}(H)$. If $\operatorname{dim} H=n$ is finite, then the orthocomplementary map is an isomorphism between $\Gamma_{k}^{\perp}(H)$ and $\Gamma_{n-k}^{\perp}(H)$. So, we assume that $4 \leqslant 2 k \leqslant \operatorname{dim} H$ without loss of generality.

Let $X$ and $Y$ be distinct $k$-dimensional subspaces of $H$ at distance 2 in $\Gamma_{k}^{\perp}(H)$. Then $X, Y$ are adjacent and non-compatible (this case was considered in Subsection 3.1) or the distance between $X$ and $Y$ in $\Gamma_{k}(H)$ is equal to 2 and $X \cap Y$ is $(k-2)$-dimensional. The following example concerns the second case.

Example 14. Suppose that $X, Y$ are compatible $k$-dimensional subspaces of $H$ whose intersection is $(k-2)$-dimensional and consider an orthonormal basis of $H$ such that $X$ and $Y$ are spanned by subsets of this basis. There is a $k$-dimensional subspace spanned by a subset of this basis and ortho-adjacent to both $X, Y$.

In the case when $X \cap Y$ is $(k-2)$-dimensional, the existence of a $k$-dimensional subspace ortho-adjacent to both $X, Y$ does not imply that $X$ and $Y$ are compatible. We give an example for $k=2$, but the general case is similar.

Example 15. Let us take any 2-dimensional subspace $Z \subset H$. Consider a 1-dimensional subspace $P \subset Z$ and the 1-dimensional subspace $Q=Z \cap P^{\perp}$ which is the unique 1dimensional subspace of $Z$ orthogonal to $P$. Let $X$ be a 2 -dimensional subspace containing $Q$ and orthogonal to $P$ (since $\operatorname{dim} H \geqslant 4$, such a subspace is not unique). Similarly, we take a 1-dimensional subspace $P^{\prime} \subset Z$ distinct from $P, Q$, the 1-dimensional subspace $Q^{\prime}=Z \cap P^{\perp \perp}$ (which is also distinct from $P, Q$ ) and any 2-dimensional subspace $Y$ containing $Q^{\prime}$ and orthogonal to $P^{\prime}$. We can choose $X, Y$ such that $X \cap Y=0$ and $X, Y$ are non-orthogonal. Then $X, Y$ are non-compatible; on the other hand, each of $X, Y$ is ortho-adjacent to $Z$.

Lemma 16. Let $X$ and $Y$ be compatible $k$-dimensional subspaces of $H$ whose intersection is $(k-2)$-dimensional. Then for every $k$-dimensional subspace $Z \subset H$ ortho-adjacent to both $X, Y$ there are precisely two $k$-dimensional subspaces of $H$ ortho-adjacent to each of $X, Y, Z$.

Proof. Since $X, Y, Z$ are mutually compatible, there is an orthonormal basis $B$ of $H$ such that each of these subspaces is spanned by a subset of $B$. Observe that the $(k-2)$ dimensional subspace $X \cap Y$ and the 2-dimensional subspaces

$$
X^{\prime}=X \cap(X \cap Y)^{\perp} \quad \text { and } \quad Y^{\prime}=Y \cap(X \cap Y)^{\perp}
$$


are also spanned by subsets of $B$. Since $X, Z, Y$ is a geodesic in $\Gamma_{k}^{\perp}(H)$, the subspace $Z$ contains $X \cap Y$ and intersects $X^{\prime}$ and $Y^{\prime}$ in 1-dimensional subspaces $P$ and $Q$, respectively. Denote by $P^{\prime}$ and $Q^{\prime}$ the 1-dimensional subspaces which are the orthogonal complements of $P$ and $Q$ in $X^{\prime}$ and $Y^{\prime}$, respectively. Each of the 1-dimensional subspaces $P, P^{\prime}, Q, Q^{\prime}$ contains a vector from $B$. The $k$-dimensional subspaces

$$
Z_{1}=P^{\prime}+(X \cap Y)+Q \quad \text { and } \quad Z_{2}=P+(X \cap Y)+Q^{\prime}
$$

are ortho-adjacent to $X, Y, Z$ and spanned by subsets of $B$.

Suppose that $Z^{\prime}$ is a $k$-dimensional subspace ortho-adjacent to $X, Y, Z$. Then subspaces $X, Y, Z, Z^{\prime}$ are mutually compatible and there is an orthonormal basis $B^{\prime}$ such that each of these subspaces is spanned by a subset of $B^{\prime}$. The subspaces $X \cap Y, X^{\prime}, Y^{\prime}$ are also spanned by vectors from $B^{\prime}$ and each of the 1-dimensional subspaces $P, P^{\prime}, Q, Q^{\prime}$ contains a vector from $B^{\prime}$. As above, we establish that $Z^{\prime}$ contains $X \cap Y$, the subspaces $X^{\prime} \cap Z^{\prime}, Y^{\prime} \cap Z^{\prime}$ are 1-dimensional and

$$
Z^{\prime}=\left(X^{\prime} \cap Z^{\prime}\right)+(X \cap Y)+\left(Y^{\prime} \cap Z^{\prime}\right)
$$

Since $X^{\prime} \cap Z^{\prime}$ is a 1-dimensional subspace of $X^{\prime}$ containing a vector from $B^{\prime}$, it coincides with $P$ or $P^{\prime}$. Similarly, $Y^{\prime} \cap Z^{\prime}$ coincides with $Q$ or $Q^{\prime}$. Since $Z$ and $Z^{\prime}$ are ortho-adjacent, we obtain that $Z^{\prime}$ is $Z_{1}$ or $Z_{2}$.

Lemma 17. Suppose that $X$ and $Y$ are $k$-dimensional subspaces of $H$ whose intersection is $(k-2)$-dimensional and there are two ortho-adjacent $k$-dimensional subspaces $Z, Z^{\prime} \subset H$ such that each of these subspaces is ortho-adjacent to both $X, Y$. Then $X$ and $Y$ are compatible.

Proof. First, we show that the $(k-1)$-dimensional subspace $Z \cap Z^{\prime}$ is contained in $X$ or $Y$. If $Z \cap Z^{\prime}$ is not contained in $X$, then $Z$ and $Z^{\prime}$ intersect $X$ in distinct $(k-1)$ dimensional subspaces whose sum coincides with $X$. Similarly, $Z \cap Z^{\prime} \not \subset Y$ implies that $Z$ and $Z^{\prime}$ intersect $Y$ in distinct $(k-1)$-dimensional subspaces whose sum is $Y$. Then the $(k+1)$-dimensional subspace $Z+Z^{\prime}$ contains both $X, Y$ and $\operatorname{dim}(X+Y)<k+2$ which contradicts the assumption that $X \cap Y$ is $(k-2)$-dimensional.

Without loss of generality, we can assume that $Z \cap Z^{\prime}$ is a $(k-1)$-dimensional subspace of $X$. We have

$$
\operatorname{dim}(X \cap Y)=k-2 \text { and } \operatorname{dim}(X+Y)=k+2,
$$

which means that the orthogonal complement of $X \cap Y$ in $X+Y$ is 4-dimensional. We denote this subspace by $M$. The subspaces

$$
X^{\prime}=X \cap M \quad \text { and } \quad Y^{\prime}=Y \cap M
$$

are 2-dimensional. Since $X, Z, Y$ and $X, Z^{\prime}, Y$ are geodesics in $\Gamma_{k}^{\perp}(H)$, each of $Z, Z^{\prime}$ contains $X \cap Y$ and is contained in $X+Y$ which implies that

$$
S=Z \cap M \quad \text { and } \quad S^{\prime}=Z^{\prime} \cap M
$$


are distinct 2-dimensional subspaces. Recall that $Z \cap Z^{\prime}$ is a $(k-1)$-dimensional subspace of $X$ containing $X \cap Y$, and consequently,

$$
Z \cap Z^{\prime} \cap M=S \cap S^{\prime}
$$

is a 1-dimensional subspace of $X^{\prime}$.

Our next step is to show that $S$ and $S^{\prime}$ are compatible to both $X^{\prime}, Y^{\prime}$. We will use the following fact [9, Lemma 1.14]: if a closed subspace $A \subset H$ is compatible to closed subspaces $B, C \subset H$, then $A$ is also compatible to $B \cap C, \overline{B+C}$ and $B^{\perp}$. Since $Z$ is compatible to $X \cap Y$ and $X+Y$, it is compatible to

$$
M=(X+Y) \cap(X \cap Y)^{\perp} .
$$

Then $Z$ is compatible to $X^{\prime}=X \cap M$. So, $X^{\prime}$ is compatible to $Z$ and $M$ (the latter follows from the fact that $\left.X^{\prime} \subset M\right)$ and, consequently, it is compatible to $S=Z \cap M$. Similarly, we establish that $X^{\prime}$ is compatible to $S^{\prime}$ and $Y^{\prime}$ is compatible to both $S, S^{\prime}$.

Using the fact that $S, S^{\prime}$ are compatible to both $X^{\prime}, Y^{\prime}$, we establish that $X^{\prime}$ and $Y^{\prime}$ are orthogonal which immediately implies that $X$ and $Y$ are compatible.

It was noted above that $S \cap S^{\prime}$ is a 1-dimensional subspace of $X^{\prime}$. We denote this subspace by $P$ and write $Q$ for the unique 1-dimensional subspace of $X^{\prime}$ orthogonal to $P$. Since $X^{\prime}$ and $S$ are compatible 2-dimensional subspaces, $Q$ is orthogonal to $S$. Similarly, it is orthogonal to $S^{\prime}$. Then $Q$ is orthogonal to $S+S^{\prime}$. Since

$$
Z=(X \cap Y)+S \quad \text { and } \quad Z^{\prime}=(X \cap Y)+S^{\prime}
$$

are ortho-adjacent to $Y=(X \cap Y)+Y^{\prime}$, the subspaces $S$ and $S^{\prime}$ intersect $Y^{\prime}$ in 1dimensional subspaces $P_{1}$ and $P_{2}$, respectively. These subspaces are distinct (otherwise, they coincide with $S \cap S^{\prime}$ and $X^{\prime} \cap Y^{\prime} \neq 0$ which is impossible). Therefore, $Y^{\prime}=P_{1}+P_{2}$ is contained in $S+S^{\prime}$, and consequently, $Q$ is orthogonal to $Y^{\prime}$.

Now, we show that $P$ is orthogonal to $Y^{\prime}$. Denote by $Q_{i}$ the 1-dimensional subspace of $Y^{\prime}$ orthogonal to $P_{i}, i=1,2$. We have $Q_{1} \neq Q_{2}$, since $P_{1} \neq P_{2}$. Then $Q_{1}$ is orthogonal to $S$ (since $S$ and $Y^{\prime}$ are compatible) and, for the same reasons, $Q_{2}$ is orthogonal to $S^{\prime}$. This implies that both $Q_{1}, Q_{2}$ are orthogonal to $P=S \cap S^{\prime}$, and hence, $Y^{\prime}=Q_{1}+Q_{2}$ is orthogonal to $P$. Then $X^{\prime}=P+Q$ is orthogonal to $Y^{\prime}$ and we get the claim.

By assumption, we have $4 \leqslant 2 k \leqslant \operatorname{dim} H$ and distinguish the following three cases:

- $k \leqslant \operatorname{dim} H-4$ which means that $k \geqslant 4$ or $k=3, \operatorname{dim} H \geqslant 7$ or $k=2, \operatorname{dim} H \geqslant 6$.

- $k=\operatorname{dim} H-3$ which means that $\operatorname{dim} H=2 k=6$ or $k=2, \operatorname{dim} H=5$.

- $k=\operatorname{dim} H-2$ which means that $\operatorname{dim} H=2 k=4$.

We need the following clarification of Lemma 9.

Lemma 18. For non-compatible adjacent $k$-dimensional subspaces $X, Y \subset H$ the following assertions are fulfilled: 
(1) If $k \leqslant \operatorname{dim} H-4$, then there are infinitely many $k$-dimensional subspaces $Z \subset H$ ortho-adjacent to both $X, Y$ and such that there are infinitely many $k$-dimensional subspaces $Z^{\prime} \subset H$ ortho-adjacent to $X, Y, Z$.

(2) If $k=\operatorname{dim} H-3$, then there are infinitely many $k$-dimensional subspaces $Z \subset H$ ortho-adjacent to both $X, Y$ and such that there is precisely one $Z^{\prime} \subset H$ ortho-adjacent to $X, Y, Z$.

(3) If $\operatorname{dim} H=2 k=4$, then there are precisely two 2-dimensional subspaces orthoadjacent to both $X, Y$; these subspaces are orthogonal.

Proof. (1). Since $k \leqslant \operatorname{dim} H-4$ and $X+Y$ is $(k+1)$-dimensional, we have

$$
\operatorname{dim}(X+Y)^{\perp} \geqslant 3
$$

and for every 1-dimensional subspace $P \subset(X+Y)^{\perp}$ there are infinitely many 1-dimensional subspaces $Q \subset(X+Y)^{\perp}$ orthogonal to $P$. For any such $P$ and $Q$ the $k$-dimensional subspaces

$$
P+(X \cap Y), Q+(X \cap Y), X, Y
$$

are mutually ortho-adjacent.

(2). When $k=\operatorname{dim} H-3$, then the subspace $(X+Y)^{\perp}$ is 2-dimensional and for any 1-dimensional subspace $P \subset(X+Y)^{\perp}$ there is a unique 1-dimensional subspace $Q \subset(X+Y)^{\perp}$ orthogonal to $P$. As above, the $k$-dimensional subspaces (6) are mutually ortho-adjacent. The $k$-dimensional subspace $S+W$, where $S$ is the orthogonal complement of $X \cap Y$ in $X+Y$ and $W$ is a $(k-2)$-dimensional subspace of $X \cap Y$ (see Lemma 7 ), is not adjacent to $P+(X \cap Y)$. So, $Q+(X \cap Y)$ is the unique $k$-dimensional subspace ortho-adjacent to $X, Y, P+(X \cap Y)$.

(3). Suppose that $\operatorname{dim} H=2 k=4$. By Lemma 9, there are precisely two 2-dimensional subspaces ortho-adjacent to both $X, Y$ : one of them is the sum of $X \cap Y$ and $(X+Y)^{\perp}$ and the second is the orthogonal complement of $X \cap Y$ in $X+Y$.

For $k \leqslant \operatorname{dim} H-3$ we can characterise adjacency in terms of ortho-adjacency.

Lemma 19. Let $X$ and $Y$ be $k$-dimensional subspaces of $H$ such that the distance between $X, Y$ in $\Gamma_{k}^{\perp}(H)$ is equal to 2 . Then the following assertions are fulfilled:

(1) In the case when $k \leqslant \operatorname{dim} H-4$, the subspaces $X, Y$ are adjacent and non-compatible if and only if there are infinitely many $k$-dimensional subspaces $Z \subset H$ ortho-adjacent to both $X, Y$ and such that there are infinitely many $k$-dimensional subspaces $Z^{\prime} \subset H$ ortho-adjacent to $X, Y, Z$.

(2) In the case when $k=\operatorname{dim} H-3$, the subspaces $X, Y$ are adjacent and non-compatible if and only if there are infinitely many $k$-dimensional subspaces $Z \subset H$ ortho-adjacent to both $X, Y$ and such that there is precisely one $Z^{\prime} \subset H$ ortho-adjacent to $X, Y, Z$. 
Proof. (1). If $X, Y$ are adjacent and non-compatible, then the required condition holds by the statement (1) of Lemma 18. Conversely, suppose that there are infinitely many $k$-dimensional subspaces $Z \subset H$ ortho-adjacent to both $X, Y$ and such that there are infinitely many $k$-dimensional subspaces $Z^{\prime} \subset H$ ortho-adjacent to $X, Y, Z$. If $X, Y$ are not adjacent, then their intersection is $(k-2)$-dimensional. Since there is a pair of ortho-adjacent $k$-dimensional subspaces of $H$ which are ortho-adjacent to both $X, Y$, Lemma 17 implies that $X$ and $Y$ are compatible. Then, by Lemma 16, for every $k$ dimensional subspace $Z \subset H$ ortho-adjacent to both $X, Y$ there are precisely two $k$ dimensional subspaces of $H$ ortho-adjacent to $X, Y, Z$ which contradicts our assumption. So, $X, Y$ are adjacent. Since the distance between $X$ and $Y$ in $\Gamma_{k}^{\perp}(H)$ is equal to 2, they are non-compatible.

(2). We use the same arguments and the statement (2) of Lemma 18 instead of the statement (1).

Now, we can prove Theorem 3. Let $f$ be an automorphism of $\Gamma_{k}^{\perp}(H)$. If $k \geqslant 3$ or $\operatorname{dim} H \geqslant 5$ and $k=2$, then $f$ is an automorphism of $\Gamma_{k}(H)$ by Lemma 19 and the required statement follows from Lemma 11.

Remark 20. Suppose that $\operatorname{dim} H=2 k=4$. In terms of ortho-adjacency, as it is seen by Example 5, the adjacency relation cannot be characterised while the orthogonality relation can be as follows: 2-dimensional subspaces $X, Y \subset H$ are orthogonal if and only if for every 2-dimensional subspace $Z \subset H$ ortho-adjacent to both $X, Y$ there are precisely two 2-dimensional subspaces of $H$ ortho-adjacent to $X, Y, Z$ (we use Lemma 16 and arguments from the proof of Lemma 19). Therefore, if $\operatorname{dim} H=4$, then every automorphism $f$ of $\Gamma_{2}^{\perp}(H)$ is orthogonality preserving in both directions; since two 2-dimensional subspaces of $H$ are compatible if and only if they are ortho-adjacent or orthogonal, $f$ is compatible preserving in both directions. Compatibility preserving transformations of Hilbert Grassmannians are determined except the case when $\operatorname{dim} H=2 k$ is equal 4 or 6 (see [9, Chapter 5]).

Remark 21. Let $V$ be a vector space (over a field) equipped with an anisotropic symmetric form. The ortho-Grassmann graph formed by 2-dimensional subspaces of $V$ is investigated in [5]. If $\operatorname{dim} V \geqslant 5$, then every graph automorphism is induced by an orthogonality preserving semilinear automorphism of $V$. The main result of [5] concerns the case when $\operatorname{dim} V=4$. As a simple consequence, we obtain the following: if $V$ is a real Hilbert space of dimension 4 and $f$ is a graph automorphism, then there is an orthogonal operator $O$ such that for every 2-dimensional subspace $X \subset H$ we have $f(X)=O(X)$ or $f(X)=O(X)^{\perp}$. Algebraic arguments used in [5] do not work in our case (the inner product on complex Hilbert spaces is Hermitian).

\section{Generalised ortho-Grassmann graphs associated to conjugacy classes of finite-rank self-adjoint operators}

Recall that two operators $A$ and $B$ on $H$ are unitary conjugate if there is a unitary operator $U$ on $H$ such that $B=U A U^{*}$. For example, any two rank- $m$ projections are 
unitary conjugate and $\mathcal{P}_{m}(H)$ is the conjugacy class formed by all such projections. Let $\mathcal{C}$ be a conjugacy class consisting of finite-rank self-adjoint operators on $H$. This class is completely determined by the spectrum $\sigma=\left\{a_{1}, \ldots, a_{k}\right\}$ of operators from $\mathcal{C}$ (each eigenvalue $a_{i}$ is real) and the set $d=\left\{n_{1}, \ldots, n_{k}\right\}$, where $n_{i}$ is the dimension of the eigenspaces corresponding to the eigenvalue $a_{i}$. The eigenspaces of every operator from $\mathcal{C}$ are mutually orthogonal, their sum is $H$ and the eigenspace corresponding to every non-zero $a_{i}$ is finite-dimensional. If one of $a_{i}$ is zero, then the kernels of operators from $\mathcal{C}$ are non-trivial. If $H$ is infinite-dimensional, then $0 \in \sigma$ and the kernels are infinitedimensional (since operators from $\mathcal{C}$ are of finite rank and their kernels are the orthogonal complements of the images).

In what follows, the conjugacy class $\mathcal{C}$ will be denoted by $\mathcal{G}(\sigma, d)$ and called the $(\sigma, d)$ Grassmannian. For example, if $\sigma=\{0, \lambda\}$, where $\lambda$ is a non-zero real number, and $d=\{\operatorname{dim} H-m, m\}$, then $\mathcal{G}(\sigma, d)$ is the conjugacy class $\lambda \mathcal{P}_{m}(H)$ formed by all $\lambda$-multiples of rank- $m$ projections. If $\operatorname{dim} H=n$ is finite and $d=\{n\}$, then $\mathcal{G}(\sigma, d)$ consists of a unique operator which is a scalar multiple of the identity.

If $A \in \mathcal{G}(\sigma, d)$ and $X_{i}$ is the eigenspace of $A$ corresponding to $a_{i}$, then

$$
A=\sum_{i=1}^{k} a_{i} P_{X_{i}} .
$$

Let $S(d)$ be the group of all permutations $\delta$ on $\{1, \ldots, k\}$ such that $n_{\delta(i)}=n_{i}$ (the group is trivial if all $n_{i}$ are mutually distinct). For every permutation $\delta \in S(d)$ the operator

$$
\delta(A)=\sum_{i=1}^{k} a_{i} P_{X_{\delta(i)}}
$$

belongs to $\mathcal{G}(\sigma, d)$. For example, if $\operatorname{dim} H=2 m, \sigma=\{0,1\}$ and $d=\{m, m\}$, then $\mathcal{G}(\sigma, d)=\mathcal{P}_{m}(H)$ and $S(d)$ coincides with $S_{2}$; furthermore, if $\delta$ is the non-trivial element of $S(d)$, then for every projection $P_{X} \in \mathcal{P}_{m}(H)$ we have $\delta\left(P_{X}\right)=P_{X^{\perp}}$.

Operators $A, B \in \mathcal{G}(\sigma, d)$ are called adjacent if the following conditions are satisfied:

(A1) the rank of $A-B$ is equal to 2 ,

(A2) the kernel and the image of $A-B$ are invariant to both $A, B$;

see [10] for the details. This concept admits a simple interpretation in terms of eigenspaces. If $H$ is infinite-dimensional, then $0 \in \sigma$ and the kernels of operators from $\mathcal{G}(\sigma, d)$ are infinite-dimensional, but their codimension is finite. Two closed subspaces of the same finite codimension are called adjacent if their orthogonal complements are adjacent, and we say that these subspaces are ortho-adjacent if the orthogonal complements are orthoadjacent. For every $i \in\{1, \ldots, k\}$ denote by $X_{i}$ and $Y_{i}$ the eigenspaces of $A$ and $B$ (respectively) corresponding to the eigenvalue $a_{i}$. Then $A$ and $B$ are adjacent if and only if there are distinct $i, j \in\{1, \ldots, k\}$ such that the following assertions are fulfilled: 
- $X_{i}$ and $X_{j}$ are adjacent to $Y_{i}$ and $Y_{j}$, respectively ${ }^{1}$;

- $X_{t}=Y_{t}$ for all $t \neq i, j$, and consequently, $X_{i}+X_{j}=Y_{i}+Y_{j}$.

In this case, the operators $A, B$ are said to be $(i, j)$-adjacent. For example, rank-m projections $P_{X}$ and $P_{Y}$ are adjacent if and only if the $m$-dimensional subspaces $X$ and $Y$ are adjacent; furthermore, $P_{X}$ and $P_{Y}$ are adjacent and commute if and only if $X$ and $Y$ are ortho-adjacent. If $A$ and $B$ commute, then each of these operators commute with $A-B$ which immediately implies (A2). We say that $A, B$ are commutatively adjacent if they commute and satisfy (A1). This means that $A, B$ are $(i, j)$-adjacent for some distinct $i, j \in\{1, \ldots, k\}$; furthermore, $X_{i}$ and $X_{j}$ are ortho-adjacent to $Y_{i}$ and $Y_{j}$, respectively (it is well-known that two compact self-adjoint operators commute if and only if their eigenspaces are mutually compatible).

The generalised Grassmann graph $\Gamma(\sigma, d)$ is the simple graph whose vertex set is $\mathcal{G}(\sigma, d)$ and two operators are connected by an edge if they are adjacent. If $\mathcal{G}(\sigma, d)=\mathcal{P}_{m}(H)$, then $\Gamma(\sigma, d)=\Gamma_{m}(H)$. The graph $\Gamma(\sigma, d)$ is connected [10]. Any other $\Gamma\left(\sigma^{\prime}, d\right)$ is isomorphic to $\Gamma(\sigma, d)$. Indeed, if $\sigma^{\prime}=\left\{a_{1}^{\prime}, \ldots, a_{k}^{\prime}\right\}$, then the correspondence

$$
\sum_{i=1}^{k} a_{i} P_{X_{i}} \rightarrow \sum_{i=1}^{k} a_{i}^{\prime} P_{X_{i}}
$$

defines an isomorphism between these graphs. In particular, if $k=2$ (i.e. there are precisely two eigenvalues for operators from $\mathcal{G}(\sigma, d)$ ), then $\Gamma(\sigma, d)$ can be identified with the Grassmann graph $\Gamma_{m}(H)$, where $m$ is $n_{1}$ or $n_{2}$ (at least one of $n_{1}, n_{2}$ is finite). For every unitary or anti-unitary operator $U$ on $H$ the transformation sending every $A \in$ $\mathcal{G}(\sigma, d)$ to $U A U^{*}$ is an automorphism of $\Gamma(\sigma, d)$ preserving each type of adjacency and for every non-identity $\delta \in S(d)$ the transformation sending every $A \in \mathcal{G}(\sigma, d)$ to $\delta(A)$ is an automorphism of $\Gamma(\sigma, d)$ which does not preserve all types of adjacency. The following is an analogue of Chows's theorem concerning automorphisms of $\Gamma(\sigma, d)$.

Theorem 22 ([10]). Suppose that $k \geqslant 3$ and $n_{i}>1$ for all $i \in\{1, \ldots, k\}$. Then for every automorphism $f$ of $\Gamma(\sigma, d)$ there are a unitary or anti-unitary operator $U$ on $H$ and a permutation $\delta \in S(d)$ such that

$$
f(A)=U \delta(A) U^{*}
$$

for all $A \in \mathcal{G}(\sigma, d)$. In particular, every automorphism of $\Gamma(\sigma, d)$ preserving each type of adjacency is induced by a unitary or anti-unitary operator on $H$.

Remark 23. The above statement fails for $k=2$ (since $\Gamma(\sigma, d)$ is identified with $\Gamma_{m}(H)$, $\left.m \in\left\{n_{1}, n_{2}\right\}\right)$.

\footnotetext{
${ }^{1}$ Since $X_{j}$ and $Y_{j}$ are the orthogonal complements of $X_{i}$ and $Y_{i}$ (respectively) in $X_{i}+X_{j}=Y_{i}+Y_{j}$, the subspaces $X_{i}$ and $Y_{i}$ are adjacent if and only if $X_{j}$ and $Y_{j}$ are adjacent.
} 
Denote by $\Gamma^{c}(\sigma, d)$ the simple graph whose vertex set is $\mathcal{G}(\sigma, d)$ and two operators are connected by an edge if they are commutatively adjacent. Then $\Gamma^{c}(\sigma, d)=\Gamma_{m}^{\perp}(H)$ if $\mathcal{G}(\sigma, d)=\mathcal{P}_{m}(H)$. Any other $\Gamma^{c}\left(\sigma^{\prime}, d\right)$ is isomorphic to $\Gamma^{c}(\sigma, d)$ (if $\sigma^{\prime}=\left\{a_{1}^{\prime}, \ldots, a_{k}^{\prime}\right\}$, then (7) is a graph isomorphism). If $k=2$, then $\Gamma^{c}(\sigma, d)$ is identified with the ortho-Grassmann graph $\Gamma_{m}^{\perp}(H)$, where $m$ is $n_{1}$ or $n_{2}$.

Proposition 24. The graph $\Gamma^{c}(\sigma, d)$ is connected.

Proof. Proposition 1 gives the claim for $k=2$. Suppose that $k \geqslant 3$ and $A, B \in \mathcal{G}(\sigma, d)$ are non-commutative and $(i, j)$-adjacent for some $i, j \in\{1, \ldots, k\}$. For every $t \in\{1, \ldots, k\}$ denote by $X_{t}$ and $Y_{t}$ the eigenspaces of $A$ and $B$ (respectively) corresponding to $a_{t}$. Then $X_{t}=Y_{t}$ for all $t \neq i, j$ and $X_{i}, X_{j}$ are adjacent to $Y_{i}, Y_{j}$, respectively. We write $V$ for the $\left(n_{i}+n_{j}\right)$-dimensional subspace $X_{i}+X_{j}=Y_{i}+Y_{j}$ and take any $n_{i}$-dimensional subspace $Z_{i} \subset V$ ortho-adjacent to both $X_{i}, Y_{i}$. Let $Z_{j}$ be the orthogonal complement of $Z_{i}$ in $V$. The $n_{2}$-dimensional subspace $Z_{j}$ is ortho-adjacent to both $X_{j}, Y_{j}$. Consider the operator $C \in \mathcal{G}(\sigma, d)$ whose eigenspace corresponding to $a_{t}$ with $t \neq i, j$ is $X_{t}=Y_{t}$ and the eigenspaces corresponding to $a_{i}$ and $a_{j}$ are $Z_{i}$ and $Z_{j}$, respectively. This operator is commutatively $(i, j)$-adjacent to both $A, B$. The connectedness of $\Gamma^{c}(\sigma, d)$ follows from the fact that $\Gamma(\sigma, d)$ is connected.

Theorem 3 can be generalised as follows.

Theorem 25. Suppose that $k \geqslant 2$ and $n_{i}>1$ for all $i \in\{1, \ldots, k\}$. Also, we require that there is at most one $i$ such that $n_{i}=2$. Then for every automorphism $f$ of $\Gamma^{c}(\sigma, d)$ there are a unitary or anti-unitary operator $U$ on $H$ and a permutation $\delta \in S(d)$ such that

$$
f(A)=U \delta(A) U^{*}
$$

for all $A \in \mathcal{G}(\sigma, d)$.

If $k=2$, then $\Gamma^{c}(\sigma, d)$ is identified with $\Gamma_{m}^{\perp}(H), m \in\left\{n_{1}, n_{2}\right\}$ and Theorem 25 follows from Theorem 3 (see Remark 4). If $k=2$ and $n_{1}=n_{2}=2$, then $\operatorname{dim} H=n_{1}+n_{2}=4$, the graph $\Gamma^{c}(\sigma, d)$ is identified with $\Gamma_{2}^{\perp}(H)$ and the statement fails by Example 5 .

We prove Theorem 25 for $k \geqslant 3$ in the next section. We will use a modification of arguments from Section 4 to investigate geodesics of length 2 in $\Gamma^{c}(\sigma, d)$.

\section{Proof of Theorem 25}

Suppose that $k \geqslant 3, n_{i}>1$ for all $i \in\{1, \ldots, k\}$ and there is at most one $i$ such that $n_{i}=2$.

Let $A$ and $B$ be operators from $\mathcal{G}(\sigma, d)$ such that the distance between them in $\Gamma^{c}(\sigma, d)$ is equal to 2. For every $i \in\{1, \ldots, k\}$ we denote by $X_{i}$ and $Y_{i}$ the eigenspaces of $A$ and $B$ (respectively) corresponding to the eigenvalue $a_{i}$. Consider any $C \in \mathcal{G}(\sigma, d)$ commutatively adjacent to both $A, B$. There are precisely two eigenspaces of $C$ distinct from the corresponding eigenspaces of $A$ and the same holds for $B$. Therefore, there are at most four indices $i$ such that $X_{i} \neq Y_{i}$ (we have $X_{i} \neq Y_{i}$ for at least two $i$, otherwise $A=B$ ).

We start from the case when $X_{i} \neq Y_{i}$ for precisely two indices $i$. 
Lemma 26. Suppose that $X_{i} \neq Y_{i}, X_{j} \neq Y_{j}$, and $X_{t}=Y_{t}$ for $t \neq i, j$. If $C \in \mathcal{G}(\sigma, d)$ is commutatively adjacent to both $A, B$, then it is commutatively $(i, j)$-adjacent to $A$ and $B$.

Proof. For every $t \in\{1, \ldots, k\}$ we denote by $Z_{t}$ the eigenspace of $C$ corresponding to $a_{t}$. Suppose that $A, C$ are commutatively $\left(i^{\prime}, j^{\prime}\right)$-adjacent and $\{i, j\} \neq\left\{i^{\prime}, j^{\prime}\right\}$. If we have $\{i, j\} \cap\left\{i^{\prime}, j^{\prime}\right\}=\emptyset$, then

$$
Z_{i}=X_{i} \neq Y_{i}, \quad Z_{j}=X_{j} \neq Y_{j}, \quad Z_{i^{\prime}} \neq X_{i^{\prime}}=Y_{i^{\prime}}, \quad Z_{j^{\prime}} \neq X_{j^{\prime}}=Y_{j^{\prime}}
$$

that is $Z_{t} \neq Y_{t}$ for four indices $t$ which means that $B, C$ are not adjacent. Therefore, $\{i, j\} \cap\left\{i^{\prime}, j^{\prime}\right\}$ is one-element. Without loss of generality we assume that $i \neq i^{\prime}$ and $j=j^{\prime}$, i.e. $A, C$ are commutatively $\left(i^{\prime}, j\right)$-adjacent. Then

$$
Z_{i^{\prime}} \neq X_{i^{\prime}}=Y_{i^{\prime}} \quad \text { and } \quad Z_{i}=X_{i} \neq Y_{i}
$$

and, since $B, C$ are commutatively adjacent, they are commutatively $\left(i, i^{\prime}\right)$-adjacent.

So, $A, C$ are commutatively $\left(i^{\prime}, j\right)$-adjacent and $B, C$ are commutatively $\left(i, i^{\prime}\right)$-adjacent. This implies that $X_{i}=Z_{i}$ is ortho-adjacent to $Y_{i}$ and $X_{j}$ is ortho-adjacent to $Z_{j}=$ $Y_{j}$. Then $A, B$ are commutatively $(i, j)$-adjacent which contradicts our assumption.

As in Lemma 26, we suppose that $X_{i} \neq Y_{i}, X_{j} \neq Y_{j}$ and $X_{t}=Y_{t}$ if $t \neq i, j$. For every $C \in \mathcal{G}(\sigma, d)$ commutatively adjacent to both $A, B$ the eigenspaces corresponding to $a_{i}$ and $a_{j}$ are ortho-adjacent to $X_{i}, Y_{i}$ and $X_{j}, Y_{j}$ (respectively) and the eigenspace corresponding to $a_{t}, t \neq i, j$ coincides with $X_{t}=Y_{t}$. Denote by $V_{i j}$ the $\left(n_{i}+n_{j}\right)$-dimensional subspace $X_{i}+X_{j}=Y_{i}+Y_{j}$. Recall that at least one of $n_{i}, n_{j}$ is finite. If $n_{i}$ is finite, then the distance between $X_{i}$ and $Y_{i}$ in $\Gamma_{n_{i}}^{\perp}\left(V_{i j}\right)$ is equal to 2 and one of the following possibilities is realised:

(1) $X_{i}, Y_{i}$ are adjacent and non-compatible,

(2) $\operatorname{dim}\left(X_{i} \cap Y_{i}\right)=n_{i}-2$.

Since $X_{j}, Y_{j}$ are the orthogonal complements of $X_{i}, Y_{i}$ in $V_{i j}$, the same holds for the index $j$ (if $n_{j}=\infty$, then the possibility (2) is written as follows: the codimension of $X_{j} \cap Y_{j}$ in both $X_{j}, Y_{j}$ is equal to 2). In the case (1), the operators $A, B$ are non-commutative and adjacent. Since both $n_{i}, n_{j}$ are not less than 2 and at least one of them is not less than 3 , we have

$$
\operatorname{dim} V_{i j}=n_{i}+n_{j} \geqslant 5
$$

and

$$
\min \left\{n_{i}, n_{j}\right\} \leqslant n_{i}+n_{j}-3
$$

Lemma 19 implies the following.

Lemma 27. If $X_{i} \neq Y_{i}, X_{j} \neq Y_{j}$ and $X_{t}=Y_{t}$ for $t \neq i, j$, then the following assertions are fulfilled: 
(1) In the case when $\min \left\{n_{i}, n_{j}\right\} \leqslant n_{i}+n_{j}-4$, the operators $A, B$ are adjacent and non-commutative if and only if there are infinitely many $C \in \mathcal{G}(\sigma, d)$ commutatively adjacent to both $A, B$ and such that there are infinitely many $C^{\prime} \in \mathcal{G}(\sigma, d)$ commutatively adjacent to $A, B, C$.

(2) In the case when $\min \left\{n_{i}, n_{j}\right\}=n_{i}+n_{j}-3$, the operators $A, B$ are adjacent and non-commutative if and only if there are infinitely many $C \in \mathcal{G}(\sigma, d)$ commutatively adjacent to both $A, B$ and such that there is precisely one $C^{\prime} \in \mathcal{G}(\sigma, d)$ commutatively adjacent to $A, B, C$.

Lemma 28. If $X_{i} \neq Y_{i}$ for three or four indices $i$, then the number of operators in $\mathcal{G}(\sigma, d)$ commutatively adjacent to both $A, B$ is finite.

Proof. Suppose that $X_{i} \neq Y_{i}$ precisely for $i \in\{1,2,3,4\}$. If $C \in \mathcal{G}(\sigma, d)$ is commutatively adjacent to both $A, B$, then it is $(s, t)$-adjacent to $A$ for some $s, t \in\{1,2,3,4\}$ and $\left(s^{\prime}, t^{\prime}\right)$ adjacent to $B$ for $\left\{s^{\prime}, t^{\prime}\right\}=\{1,2,3,4\} \backslash\{s, t\}$ (otherwise there is $i \in\{1,2,3,4\}$ such that $\left.X_{i}=Y_{i}\right)$. This means that the eigenspace of $C$ corresponding to $a_{j}$ with $j \in\left\{s^{\prime}, t^{\prime}\right\}$ is $X_{j}$, the eigenspace of $C$ corresponding to $a_{j}, j \in\{s, t\}$ is $Y_{j}$ and the eigenspaces of $C$ associated to the remaining $a_{t}$ are $X_{t}=Y_{t}$. So, there are at most six operators $C \in \mathcal{G}(\sigma, d)$ commutatively adjacent to both $A, B$.

Consider the case when $X_{i} \neq Y_{i}$ precisely for $i \in\{1,2,3\}$. Suppose that $C \in \mathcal{G}(\sigma, d)$ is $(s, t)$-adjacent to $A$ and $\left(s^{\prime}, t^{\prime}\right)$-adjacent to $B$. If $t \notin\{1,2,3\}$, then the eigenspace of $C$ corresponding to $a_{t}$ is distinct from $X_{t}=Y_{t}$, and consequently, $t \in\left\{s^{\prime}, t^{\prime}\right\}$. Without loss of generality we assume that $t=t^{\prime}$. Then for $j \in\{1,2,3\} \backslash\left\{s, s^{\prime}\right\}$ we have $X_{j}=Y_{j}$ which is impossible. So, $s, s^{\prime}, t, t^{\prime}$ belong to $\{1,2,3\}$. This means that $C$ is $(s, j)$-adjacent to $B$ or $(t, j)$-adjacent to $B$ for $\{s, t, j\}=\{1,2,3\}$.

If $C$ is $(s, j)$-adjacent to $B$, then the eigenspace of $C$ corresponding to $a_{t}$ is $Y_{t}$. Since $C$ is $(s, t)$-adjacent to $A$, the eigenspace of $C$ corresponding to $a_{j}$ is $X_{j}$. Then $X_{j}, Y_{t}$ are orthogonal and the eigenspace of $C$ corresponding to $a_{s}$ is the orthogonal complement of $X_{j}+Y_{t}$ in

$$
X_{1}+X_{2}+X_{3}=Y_{1}+Y_{2}+Y_{3}
$$

Therefore, there is at most one $C \in \mathcal{G}(\sigma, d)$ which is $(s, t)$-adjacent to $A$ and $(s, j)$-adjacent to $B$. Similarly, there exists at most one $C \in \mathcal{G}(\sigma, d)$ which is $(s, t)$-adjacent to $A$ and $(t, j)$-adjacent to $B$.

Since there are precisely three distinct 2 -element subsets of $\{1,2,3\}$, we can get at most six operators $C \in \mathcal{G}(\sigma, d)$ commutatively adjacent to both $A, B$.

Combining Lemmas 27 and 28, we obtain the following characterisation of adjacency in terms of commutative adjacency.

Lemma 29. Suppose that the distance between $A, B \in \mathcal{G}(\sigma, d)$ in $\Gamma^{c}(\sigma, d)$ is equal to 2. Then $A, B$ are adjacent and non-commutative if and only if one of the following possibilities is realized:

(1) There are infinitely many $C \in \mathcal{G}(\sigma, d)$ commutatively adjacent to both $A, B$ and such that there are infinitely many $C^{\prime} \in \mathcal{G}(\sigma, d)$ commutatively adjacent to $A, B, C$. 
(2) There are infinitely many $C \in \mathcal{G}(\sigma, d)$ commutatively adjacent to both $A, B$ and such that there is precisely one $C^{\prime} \in \mathcal{G}(\sigma, d)$ commutatively adjacent to $A, B, C$.

Lemma 29 shows that every automorphism of $\Gamma^{c}(\sigma, d)$ is an automorphism of $\Gamma(\sigma, d)$ and Theorem 22 gives the claim.

\section{References}

[1] W.L. Chow, On the geometry of algebraic homogeneous spaces, Ann. of Math. 50 (1949), 32-67.

[2] J. Dieudonné, La géométrie des groupes classiques (2nd edition), Springer, 1963.

[3] G.P. Gehér, P. Šemrl, Isometries of Grassmann spaces, J. Funct. Anal. 270 (2016), 1585-1601.

[4] G.P. Gehér, Wigner's theorem on Grassmann spaces, J. Funct. Anal. 273 (2017), 2994-3001.

[5] H. Havlicek, On Plücker transformations of generalized elliptic spaces, Rend. Mat. Appl. (7) 15 (1995), 39-56.

[6] L. Molnár, Transformations on the set of all n-dimensional subspaces of a Hilbert space preserving principal angles, Comm. Math. Phys. 217 (2001), 409-421.

[7] L. Molnár, Maps on the n-dimensional subspaces of a Hilbert space preserving principal angles, Proc. Amer. Math. Soc. 136 (2008), 3205-3209.

[8] M. Pankov, Grassmannians of classical buildings, World Scientific, 2010.

[9] M. Pankov, Wigner type theorems for Hilbert Grassmannians, London Mathematical Society Lecture Note Series 460, Cambridge University Press, 2020.

[10] M. Pankov, K. Petelczyc, M. Żynel, Generalized Grassmann graphs associated to conjugacy classes of finite-rank self-adjoint operators, Linear Algebra Appl. 627 (2021), $1-23$.

[11] K. Prażmowski, M. Żynel, Orthogonality of subspaces in metric-projective geometry, Adv. Geom. 11 (2011), 103-116.

[12] U. Uhlhorn, Representation of symmetry transformations in quantum mechanics, Ark. Fys. 23 (1963), 307-340.

[13] V.S. Varadarajan, Geometry of Quantum Theory (2nd edition), Springer, 2000.

[14] Z. Wan, Geometry of Matrices, World Scientific, 1996. 